\title{
Factores implicados en la transferencia de resultados de investigación en las instituciones de educación superior
}

\author{
Involved factors in the research results transfer in higher educational institutions
}

\author{
Jackeline Macias Urrego $^{1} \quad$ Alejandro Valencia Arias ${ }^{2} \quad$ Iván Montoya Restrepo $^{3}$
}

Recibido 3 de noviembre de 2016, aceptado 1 de agosto de 2017

Received: November 3, $2016 \quad$ Accepted: August 1, 2017

\begin{abstract}
RESUMEN
Este artículo tiene como objetivo examinar los factores implicados en la transferencia de resultados de investigación en las instituciones de educación superior (IES) a partir de sus capacidades de innovación lo que servirá como base para plantear mecanismos efectivos que fomenten estos procesos en las IES, entender las variables coyunturales en el tema e identificar líneas de investigación futuras. La metodología empleada es la revisión sistemática de literatura, mediante la definición de preguntas de investigación, estrategias de búsqueda y definición de criterios de calidad de las publicaciones, esto con el fin de caracterizar proceso de transferencia de resultados de investigación de las IES a partir de capacidades de innovación, describir las variables de estudio más representativas y los diseños metodológicos más utilizados en el estudio de esta temática. Entre los principales resultados se observa las condiciones que favorecen procesos de transferencia de resultados de investigación en las IES son las alianzas estratégicas, la expansión en el mercado, un mayor conocimiento de los competidores, la innovación y el aprendizaje organizacional, la inversión en CTi y las acciones de apropiación de conocimiento en las organizaciones. Además, entre los factores que han dificultado un mayor desarrollo investigativo en este campo de conocimiento se encuentran: falta de apoyo a la investigación longitudinal, menor número de académicos capacitados para investigar sobre gestión tecnológica y transferencia de conocimientos, la falta de inclusión de oficinas de transferencia tecnológica orientadas a la gestión, administración y comercialización del conocimiento en el contexto universitario.
\end{abstract}

Palabras clave: Transferencia tecnológica, gestión tecnológica, instituciones de educación superior, revisión sistemática de literatura.

\section{ABSTRACT}

This article aims to examine the involved factors in the research results transfer in the Higher Educational Institutions (HEIs) from its innovation capabilities, which will serve as a basis for suggesting effective mechanisms to promote these processes in HEIs, understanding the situational variables on the issue and identifying further research. The carried out methodology is the systematic literature review, by defining research questions, searching strategies and defining criteria of quality of publications, this in order to characterize the process of research results transfer in the HEIs from innovation capabilities, describing the most representative variables and methodological study designs commonly used on the study of this

\footnotetext{
1 Departamento de Ciencias Administrativas. Instituto Tecnológico Metropolitano. Calle 73 No 76A - 354. Medellín, Colombia. E-mail: jackelinemacias@itm.edu.co

2 Departamento de Ciencias Administrativas. Instituto Tecnológico Metropolitano. Calle 73 № 76A - 354. Medellín, Colombia. E-mail: jhoanyvalencia@itm.edu.co

3 Departamento de Ingeniería de la Organización. Universidad Nacional de Colombia. Dirección postal: Carrera 80 Nº 65-223, Medellín, Colombia. E-mail: iamontoyar@unal.edu.co
} 
subject. Among the main results, it is observed that the enabling conditions for research results transfer in the HEIs are strategic alliances, market expansion, increased knowledge of competitors, innovation and organizational learning, investment in science, technology and innovation and the actions of knowledge appropriation in organizations. Furthermore, factors that have hindered further research development in this field of knowledge are: lack of support to longitudinal research, fewer trained academic members to research on technology management and knowledge transfer, the lack of inclusion of technology transfer offices oriented to management, administration and commercialization of knowledge in the university context.

Keywords: Technology transfer, technology management, higher educational institutions, systematic literature review.

\section{INTRODUCCIÓN}

La innovación y la invención marcan la madurez del proceso de transferencia, difusión y adaptación tecnológica. La capacidad innovadora no puede ser adquirida a partir de la sola experiencia del trabajo [1], es por esto que la utilización de mecanismos para transferencia de conocimiento contribuye a mejorar el desempeño de las organizaciones receptoras, generando ventaja competitiva que contribuye a mejorar la gestión de la empresa [2]. Frente al caso de las instituciones de educación superior, se hace necesaria una mejor gestión de las capacidades organizacionales que hacen posible desempeños superiores en ambientes altamente competitivos y exigentes. Adicionalmente, una preocupación central de teóricos y practicantes de la gestión tecnológica es identificar el tipo y nivel de las capacidades de innovación que se requieren para soportar sistemas exitosos de gestión de I+D+i [3].

Además, frente al tema de la tercera misión de las universidades, se plantea que la misma está relacionada de manera directa y específica con la capacidad de innovación de la organización [4],[5], lo que es posible mediante la transferencia de conocimientos, proceso que es concebido como "el movimiento y difusión de una tecnología o producto desde su invención original a un contexto económico y social diferente" [4], donde el cambio tecnológico se admite como el motor del cambio social [1]; sin embargo, aceptar que la definición de transferencia solo abarque la tecnología y la creación de productos, implica desconocer que existen otros tipos de transferencia de conocimiento, "como el tácito y otras formas del explícito, distintas a la de los artefactos y productos" [6].

Por otra parte, en la mayoría de las universidades públicas y privadas se presenta la intencionalidad de realizar transferencia de resultados de investigación; sin embargo, gran parte de las universidades públicas tiene un énfasis hacia las publicaciones y participación en eventos académicos, lo que es planificado desde la misma generación de los proyectos; en el caso de las universidades privadas hay un énfasis en estrategias de transferencia hacia otros actores del sistema, lo que se traduce en el vínculo universidad-empresa-Estado [7].

No obstante, la problemática más visible con respecto a la intencionalidad se encuentra en que muchas instituciones de educación superior, no cuentan con "información precisa sobre el tipo de conocimientos que están transfiriendo, de qué manera, a quién, con qué propósitos, y qué mecanismos de evaluación existen para determinar el impacto y retroalimentar los procesos académicos y administrativos" [5]. Esto es un claro indicio de la relación que existente entre el funcionamiento de las organizaciones, para el caso las IES, con las diferentes capacidades (dinámicas y de innovación).

En este contexto se observa que las instituciones de educación superior, productoras importantes de conocimientos no poseen mecanismos claros para realizar transferencia de resultados de investigación, mostrando la existencia de vacíos investigativos, por lo que existe la necesidad de identificar los factores que inciden en el estudio y la estructuración de estos mecanismos [8]. Teniendo clara esta necesidad, este artículo tiene como objetivo examinar los factores implicados en la transferencia de resultados de investigación (TRINV) en las IES a partir de sus capacidades de innovación lo que servirá de insumo para entender las temáticas coyunturales en el tema, identificar líneas de investigación futuras y plantear mecanismos efectivos que fomenten estos procesos en las IES. 
Para lograr este propósito, se implementa como metodología la revisión sistemática de literatura (RSL), mediante la definición de preguntas de investigación, estrategias de búsqueda y criterios de calidad; posteriormente, se presenta el análisis de resultados alrededor de las principales características del proceso de transferencia de resultados de investigación de las IES a partir de capacidades de innovación, las variables de estudio más representativas y los diseños metodológicos más utilizadas en el estudio de esta temática.

\section{METODOLOGÍA: REVISIÓN SISTEMÁTICA DE LITERATURA (RSL)}

Las revisiones sistemáticas son búsquedas en la literatura que se adhieren estrechamente a un conjunto de métodos científicos que buscan limitar el error sistemático (sesgo), principalmente por el intento de identificar, evaluar y sintetizar todos los estudios pertinentes (de cualquier diseño), con el fin de responder a una pregunta en particular (o un conjunto de preguntas) [9].

La RSL surge como mecanismo alterno a las revisiones subjetivas, ya que estas se extienden a medida que existe un gran cantidad de estudios empíricos en el área de conocimiento, lo que dificulta identificación de las principales fuentes de literatura. Por lo tanto, se plantea la necesidad de establecer estrategias objetivas y sistemáticas para contrarrestar el debate sobre la relación entre los factores y variables implicados en un tema de investigación [9], [10]. Frente a esto, la revisión sistemática puede ayudar a aclarar los planteamientos requeridos y se convertirá en un compilado de la evidencia actual sobre un suceso respectivo. Para la RSL de esta investigación se implementaron básicamente las siguientes etapas:

A. Identificación de la estrategia de búsqueda (definición de preguntas de investigación, consultas en bibliotecas, búsquedas dirigidas a la identificación de revisiones sistémicas potencialmente relevante para el estudio, utilización de diversos buscadores de prueba para identificar combinaciones de términos afines a la temática, consultas con expertos en el campo) [11].

B. Definición de una estrategia de búsqueda bibliográfica que permita la recopilación de información existente sobre el tema, buscando el mayor número de fuentes posibles de calidad, con amplios criterios de selección [12].

C. Reportar los resultados mediante resúmenes en gráficos y tablas, que den cuenta de los hallazgos obtenidos en la RSL [9].

A continuación se amplían cada una de estas etapas:

Necesidad de la revisión y preguntas de investigación que orientan a la RSL sobre el proceso de TRINV en las IES a partir de capacidades de innovación La literatura en relación con el proceso de transferencia de resultados de investigación en las IES a partir de capacidades de innovación es escasa, lo que genera incertidumbre sobre las variables que interfieren en dicho proceso; es por lo anterior, que una revisión sistemática de literatura se convierte en una opción adecuada para explorar y compilar dichas variables, evaluar su importancia, impacto y relación en el proceso de transferencia de resultados de investigación.

Para evitar riesgos y sesgos en la revisión de la información, se establecieron preguntas de investigación que orientaron la búsqueda; durante esta se detectaron los estudios primarios pertinentes, la información que sería extraída de ellos y los mecanismos que permiten realizar una síntesis adecuada del tema. Las preguntas formuladas son las siguientes:

- ¿Cuáles son las principales características del proceso de transferencia de resultados de investigación?

- ¿Cuáles son las variables de estudio más representativas al abordar la temática del proceso de transferencia de resultados de investigación en las IES a partir de sus capacidades de innovación?

\section{Definición de la estrategia de búsqueda}

La búsqueda de información debe ser rigurosa y exhaustiva, evitando incurrir en el sesgo de selección [8]. Tomando en cuenta estas consideraciones, en esta investigación se seleccionó SciVerseScopus, ya que una de las principales bases académicas para realizar estudios bibliométricos y evaluaciones de producción científica [13].

Inicialmente para identificar los términos afines a los intereses de esta investigación, se inició con 
una identificación no sistemática, llevada a cabo mediante la revisión de narrativa previa, que permitió localizar las diferentes palabras clave que debían componer la ecuación de búsqueda; para ello se realizó la recopilación de referencias bibliográficas y de información básica de títulos y términos clave, seleccionando también algunos sinónimos para garantizar una amplitud de criterios al momento de elegir los documentos que se analizarían. Dicho proceso permitió evitar sesgo y errores conceptuales en la ecuación de búsqueda utilizada.

En el proceso de construcción se determinó que las palabras clave para definir "transferencia de resultados de investigación en las IES a partir de sus capacidades de innovación" eran: research results transfer, transfer knowledge, higher education, technology transfer, innovation capacity. A partir de estas, se realizó la construcción de la ecuación de búsqueda, para la que se tuvo en cuenta un horizonte de tiempo de 10 años (2004-2014), con el fin de garantizar que la información recopilada estuviera representada por los estudios más importantes de un período cercano y actual, y desde algunas áreas de conocimiento específicas, con el fin de proporcionar al trabajo un panorama objetivo sobre los enfoques, necesidad y variables más frecuentes de la temática abordada. Al tener en cuenta lo anterior, se definió la siguiente ecuación de búsqueda de la RSL:

((TITLE-ABS-KEY (research results transfer) AND TITLE-ABS-KEY (capacity for innovation)) AND DOCTYPE (ar OR re) AND SUBJAREA(mult OR arts OR busi OR deci OR econ OR psyc OR soci) AND PUBYEAR > 2004 AND (LIMIT-TO (SUBJAREA, "BUSI") OR LIMIT-TO (SUBJAREA, "SOCI") OR LIMIT-TO (SUBJAREA, "ECON")) AND (LIMITTO(EXACTKEYWORD, "Innovation") OR LIMIT-TO (EXACTKEYWORD, "Technology transfer") OR LIMIT-TO(EXACTKEYWORD, "Absorptive capacity”) OR LIMIT-TO (EXACTKEYWORD, "Knowledge transfer") OR LIMIT-TO (EXACTKEYWORD, "Knowledge management") OR LIMIT-TO (EXACTKEYWORD "Research and development”) OR LIMIT-TO (EXACTKEYWORD, "Research"))) OR ((TITLE-ABS-KEY (knowledge transfer) AND TITLE-ABS-KEY (innovation capacity) AND TITLE-ABS-KEY (higher education)) AND DOCTYPE (ar OR re) AND SUBJAREA (mult OR arts OR busi OR deci OR econ OR psyc OR soci) AND PUBYEAR > 2004)
Posteriormente, de los resultados arrojados por la ecuación de búsqueda se tuvieron en cuenta criterios para garantizar que la consulta de las revistas científicas y, por ende, de sus artículos fuera de la calidad. Se verificaron entonces las diferentes revistas y se evaluaron mediante el portal de Scimago Journal \& Country Rank (JCR), que incluye los indicadores científicos de los países desarrollados a partir de la información contenida en Scopus [14]. Estos indicadores se pueden utilizar para evaluar y analizar los diferentes campos científicos [15].

Ahora bien, con el fin de garantizar la calidad científica de la RSL de los resultados de la ecuación de búsqueda, se plantearon los siguientes criterios de calidad para las revistas:

A. Los journal seleccionados debían estar en alguno de los cuatro cuartiles principales (Q1-A1, Q2-A2, Q3-B y Q4-C) del indicador SCImago Journal \& Country Rank (SJR).

B. Debían estar dentro de las áreas de conocimiento afines a la temática de estudio, estás son: Business, Management and Accounting, Economics, Econometrics and Finance, Social Sciences, Computer Sciences.

C. Debían tener contenidos (teóricos o prácticos) sobre la temática de estudio transferencia de resultados de investigación en la IES a partir de capacidades de innovación.

D. La ecuación de búsqueda arrojó inicialmente 27 revistas indexadas y 38 artículos; luego de analizarlos y verificar que cumplieran con los criterios de calidad, se encontraron 17 revistas indexadas y 19 artículos que cumplieron los filtros propuestos.

\section{Registro de los datos}

Con respecto a los textos, organización y automatización de la información recolectada, se construyó una base de datos con la información encontrada en Scopus y, posteriormente, se procedió a realizar un formulario en Google Drive, el que permitió un mejor acercamiento para resolver las preguntas formuladas como propósito de la revisión. Los ítemes de este formulario son:

A. Aspectos generales de la publicación (Nombre de la revista, Título del artículo, Año de publicación del artículo, Diseños metodológicos utilizados y Población analizada).

B. Identificación de las variables (se clasificaron en los siguientes 3 tipos): Transferencia, 
Capacidades y Colectivos empresariales (Empresa-Universidad-Estado).

C. Características identificadas en cada variable: Definición de la variable y tipo de medición.

\section{ANÁLISIS DE LOS RESULTADOS}

En este apartado se exponen los resultados para cada una de las preguntas de investigación formuladas como propósitos de la RSL; se especifican aspectos generales del estudio alrededor del proceso de transferencia de resultado de investigación a partir de capacidades de innovación; y se dan a conocer las principales variables que hacen parte de este proceso, a partir de los hallazgos en la literatura académica y de su frecuencia de estudio en las publicaciones analizadas.

\section{Características del proceso de TRINV en las IES a partir de capacidades de innovación formación de alianzas de $\mathbf{I}+\mathrm{D}+\mathrm{i}$}

A partir de la revisión sistemática de literatura, se resalta la formación de alianzas en el campo de estudio de transferencia de conocimiento, el que es un mecanismo utilizado por las empresas para incrementar la transferencia de conocimiento voluntaria [16]. Este acceso al conocimiento externo puede aumentar la capacidad de innovación de una empresa [17], mediante su exposición a las nuevas tecnologías, incrementando su competitividad y dotándola a la empresa de nuevas soluciones [18].

Las alianzas tecnológicas a corto plazo, y otras formas de vínculos de investigación y desarrollo (I+D) de cooperación son fuentes ideales de los flujos de conocimiento novedoso que entra a la organización. Por consiguiente, ha sido discutida la importancia de aprender de múltiples y diversos lazos que llevan información no redundante para un rendimiento innovador. Contribuciones recientes han argumentado que el acceso al flujo de conocimiento internacional es especialmente importante para las empresas, con el objetivo de aprovechar los conocimientos mediante una mirada holística. Por lo anterior, la formación de alianzas internacionales de I+D hacen parte de un canal adicional que permite el acceso al flujo de conocimiento internacional, junto con el comercio, los pagos de tecnología extranjera, las fusiones y adquisiciones [18].

Entre los ejemplos de lo anterior, se destacan Johnson Controls y Maxwell Technologies, quienes están mutuamente desarrollando baterías con electrodos de iones de litio para usar en vehículos. Por otro lado, BMW y Google, quienes están desarrollando conjuntamente innovadores sistemas de comunicación para automóviles. Algunas alianzas de investigación y desarrollo están caracterizadas por una empresa que busca absorber el conocimiento de otra sin dar el suyo; sin embargo, en alianzas de I+D simétricas, mientras cada socio intenta mantener en última instancia su distintivo y especializado conocimiento, exponer el conocimiento facilita la cooperación y en última instancia el éxito de la alianza [19].

Por consiguiente, se debe analizar la dimensión de la capacidad de difusión de las organizaciones, ya que es importante no solo por el éxito de la transferencia de conocimiento entre empresas, sino también para las alianzas I+D. El hallazgo del grado de evaluación del conocimiento base de la empresa receptora y el éxito en la transferencia de conocimiento se encuentran positivamente relacionados, indicando que la posición de la educación de acuerdo con la literatura acerca de la relación de I+D puede también ser aplicable en un contexto organizacional.

Uso de la codificación y/o personalización del conocimiento por parte de las organizaciones

Para competir, las organizaciones usan codificación y/o personalización. Una codificación estratégica permite almacenar conocimiento organizacional explícito en bases de datos, para transferirlo a alguien de la misma compañía. Este cuerpo codificado típicamente tiene múltiples índices que permiten a los usuarios localizar y reparar, de manera eficiente, el saber. Por el contrario, una estrategia de personalización que está basada en la interacción persona-persona, incrustado dentro del contexto social, permite la transferencia de conocimiento tácito. De este conocimiento tácito, las compañías coordinan y combinan los recursos en maneras de innovar [20].

A los avances que representan estos procesos, las empresas deben seguir en constante exploración, esto indica que deben tener una conducta caracterizada por búsqueda, descubrimiento, toma de riesgos e innovación. La exploración implica que el comportamiento de las empresas sea caracterizado por refinamiento, implementación, eficiencia, producción y selección. Por ejemplo, centros de 
excelencia de tecnología de la información (TI) son grupos especiales usados por las multinacionales para reunir experiencia estratégica en informática en todo el mundo [20].

Asimismo, dentro del contexto organizacional, se ha encontrado que la generación de conocimiento e intercambio del mismo, dentro de un sistema organizacional puede fortalecer y favorecer el posicionamiento de la organización en ambientes internos y externos, esto debido a que puede aportar agilidad y dinamismo en los diversos contextos organizativos. Además, facilita la exploración y explotación de iniciativas como la creación de centros de excelencia tecnológica y de innovación, así como la generación de sistemas de información que puedan ser adaptados a otras empresas y/o instituciones que requieran de soluciones ágiles y prácticas.

\section{Capacidad de absorción}

La complejidad del aprendizaje y la innovación entre las empresas para ponerse al día radica en las diferencias en la naturaleza tácita y las fuentes o contexto específico de conocimiento tecnológico. Debido a estos aspectos, es difícil, si no imposible, para las empresas beneficiarias lograr la capacidad de innovación por medio de la mera licencia o adquisición de tecnología externa. Además, estudios como el de [21] señalaron que algunas rutinas organizacionales en donde se implementa la base tecnológica y de innovación desde sus inicios tienen estructuras claras para poder realizar transferencia. Sin embargo, se presentan dificultades de transferencia con aquellas empresas que al momento de iniciar estos procesos no han tenido estructuras mínimas tecnológicas y de investigación para recibir este conocimiento externo. Por otro lado, se argumenta que la importancia de la tecnología externa en la competencia tecnológica y la capacidad de innovación dependen de si las empresas beneficiarias han relacionado el conocimiento previo o la capacidad de absorción para entender y aprovechar las oportunidades tecnológicas. Se señala además, que en la caso de I+D tiene una función de aprendizaje y ayuda a las empresas beneficiarias a afianzar sus capacidades de absorción [22].

\section{Fuentes de tecnología externa}

El conocimiento y el análisis de las fuentes de tecnología externa se abordan desde la medición de patentes [23], licenciamientos y nivel de producción de las organizaciones, lo que hace que solo se mida desde la transferencia de tecnología; no obstante, se argumenta que las fuentes de tecnología externa (FTI), por sí solas, pueden traer dificultades para las organizaciones [24], al no promover eficazmente la innovación de las empresas nacionales en materia de patentes. Asimismo, se puede afirmar que se produce un importante efecto palanca entre la I+D (investigación y desarrollo) y la FTI (fuentes de tecnología externa). Esto implica que la compra de tecnología extranjera por sí sola no conduce a resultados de innovación de una empresa, a menos que se acople con la inversión en I+D. Desde una perspectiva estratégica, las empresas pueden utilizar la tecnología extranjera, mejorando primero su capacidad de absorción y de innovación [25], que puede ser adquirida mediante sus propias actividades de investigación y desarrollo [26].

Finalmente, la transferencia de conocimiento se considera en la actualidad como un insumo fundamental para las empresas, y gana cada vez mayor reconocimiento por su papel en la capitalización y el aprovechamiento de oportunidades. Este conocimiento es producto de la contribución que hace la organización de alguna manera para fomentar la innovación, factor clave para elevar el nivel de desarrollo y la competitividad regional [27]. Por consiguiente, el creciente número de estudios analíticos acerca de la importancia del espíritu empresarial para el desarrollo regional, en conjunto con las características de manejo de ubicación, muestran cómo la creación y funcionamiento de las empresas se basan en conocimiento; poniendo así de relieve la interacción de las empresas con las universidades que están generando conocimientos, y también con los centros de investigación y el desarrollo (I+D). Por lo tanto, esta base de conocimiento surge de la cooperación entre empresas y organismos públicos de investigación.

\section{Variables de estudio más representativos en la transferencia de conocimiento, organizaciones y capacidades de innovación}

La Tabla 1 muestra los principales diseños metodológicos utilizados por los diferentes artículos analizados; algunos de ellos, cuentan con más de un diseño en sus estudios. Los resultados muestran que el diseño metodológico más utilizado corresponde al de información secundaria (45\%). 
Este tipo de investigaciones están representados en artículos de revisión rigurosa frente a temáticas referentes o implícitas en el proceso de transferencia de conocimiento, transferencia de tecnología, organizaciones y capacidades de innovación. También están representados en artículos que toman como referente estudios mundiales y regionales sobre estos mismos temas.

Tabla 1. Principales diseños metodológicos utilizados.

\begin{tabular}{|l|c|c|}
\hline \multicolumn{1}{|c|}{ Diseños } & Frecuencia & \% \\
\hline Información secundaria & 9 & 45 \\
\hline Caso de estudio & 5 & 25 \\
\hline Encuestas & 5 & 25 \\
\hline Diseño de experimentos & 1 & 5 \\
\hline
\end{tabular}

Fuente: Elaboración propia a partir de la RSL realizada.

El segundo diseño metodológico más utilizado son las encuestas (25\%); estas se basan en la recolección de información mediante un cuestionario estructurado, que es aplicado a una muestra poblacional definida previamente según la problemática investigada, permitiendo así análisis retrospectivos de etapas o situaciones características de transferencia de conocimiento y capacidades de innovación en las organizaciones El tercer diseño metodológico utilizado es el caso de estudio (25\%), que corresponde a un diseño cualitativo donde se puede evidenciar el análisis de transferencia de conocimiento de empresas del sector privado, e incluso, de empresas chinas, mexicanas, estadounidenses.

En cuanto a la investigación en este campo, se debe recalcar que a pesar de la importancia del tema, este es un aspecto poco investigado, lo que puede deberse a las siguientes razones: falta de acceso y apoyo a la investigación longitudinal, menor número de académicos capacitados para abordar el proceso investigativo sobre el tema de transferencia de conocimientos y capacidades de innovación, la falta de inclusión de OTT/OTRIS en las organizaciones donde se pueda gestionar, administrar y comercializar la transferencia de conocimiento. Es evidente entonces la necesidad de introducir análisis de las etapas y características del proceso de transferencia de resultados de investigación entre los objetos de estudio del área temática en gestión tecnológica.
Con respecto a la población muestral analizada, se recopila en la Tabla 2 observándose que la población más frecuente son las empresas. En su mayoría, los casos analizados tienen un enfoque basado en métodos transversales cuantitativos, presentando análisis retrospectivos donde la recolección de información se da por medio de encuestas, entrevistas y casos de estudio. Las instituciones de educación superior constituyen el segundo foco poblacional de análisis (25\%); estos estudios corresponden a las mismas metodologías que el análisis de las empresas, mostrando que son métodos confiables para las investigaciones. En cuanto a la tercera y cuarta opción tituladas OTRIS $(1,5 \%)$, y países $(1,5 \%)$, se refiere únicamente a estudios a partir de información secundaria y a estudios de caso, mostrando que aún no tiene tanta fuerza el análisis de transferencia de conocimiento y capacidades de innovación en estos tipos de población.

Tabla 2. Población muestral.

\begin{tabular}{|l|c|c|}
\hline \multicolumn{1}{|c|}{ Población } & Frecuencia & $\%$ \\
\hline Empresas & 13 & 45 \\
\hline $\begin{array}{l}\text { Instituciones de educación } \\
\text { superior }\end{array}$ & 5 & 25 \\
\hline OTRIS & 1 & 1,5 \\
\hline Países & 1 & 1,5 \\
\hline
\end{tabular}

Fuente: Elaboración propia a partir de la RSL realizada.

A continuación se muestran los resultados frente a las principales variables analizadas en la revisión sistemática de literatura. Estas variables fueron divididas en 3 aspectos: transferencia, capacidades y colectivos empresariales. Esta división, así como las variables previas seleccionadas para el análisis de los artículos reportados en la RSL, fueron filtradas a partir de una revisión de literatura preliminar alrededor del proceso de transferencia de resultados de investigación y capacidades de innovación, que como se había explicado anteriormente, sirvió de base para el planteamiento de las preguntas de investigación de la RSL y de la ecuación de búsqueda seleccionada.

La Tabla 3 mustra los principales aspectos de la transferencia identificados en la RSL. Se observa que la transferencia de conocimiento es la variable 
Tabla 3. Aspectos de la transferencia analizados en la RSL.

\begin{tabular}{|l|c|c|}
\hline \multicolumn{1}{|c|}{ Transferencia } & Frecuencia & $\%$ \\
\hline $\begin{array}{l}\text { Transferencia de } \\
\text { conocimiento }\end{array}$ & 16 & 80 \\
\hline Transferencia de tecnología & 4 & 20 \\
\hline
\end{tabular}

Fuente: Elaboración propia a partir de la RSL realizada.

más estudiada y analizada con $80 \%$; esta mide "la importancia de las alianzas tecnológicas a corto plazo y otras formas de vínculos de I+D” [18]. En este aspecto se plantea que las transferencias voluntarias de conocimiento y el acceso al conocimiento externo se reconocen como fuentes principales de innovación de una empresa. En este mismo sentido, la transferencia de conocimiento mide el efecto del ajuste estratégico de la capacidad innovadora, permitiendo que la organización se acople y avance en la consolidación de sus capacidades [28].

Esta variable ayuda a comprender diferentes contextos económicos [29] y sociales de las organizaciones e involucra la comprensión también del comportamiento de las instituciones de educación superior en su contexto. Frente a esto, se afirma que las organizaciones (IES, públicas y privadas) tienen información incompleta sobre la calidad de sus invenciones o productos que se desarrollan dentro de ellas, por este aspecto es que se implementan las OTRI (oficinas de transferencia de resultados de investigación), para reducir el problema de la información asimétrica [14] y asi poder controlar y transferir sus inventos.

La segunda variable más estudiada es la transferencia de tecnología (20\%). Esta variable se mide "como una forma de conocimiento, por lo que en una medida considerable, se pueden extender los argumentos a un contexto más general de la transferencia de conocimientos" [30]. Asimismo, se comprende como la forma de medir las inversiones que realizan las organizaciones a nivel interno y externo, verificando que cumplan con las condiciones de tecnología y que permitan fortalecer las capacidades de innovación. Se establece que los nichos en donde se lleva cabo la transferencia tecnológica son los institutos o centros de investigación y desarrollo en donde se utilizan los resultados de investigación de proyectos de I+D [31]; estos resultados tienen que ver desde la generación de nuevo conocimiento, desarrollo tecnológico e innovación, apropiación social del conocimiento o resultados de actividades relacionadas con la formación de recursos humanos para ciencia, tecnología e innovación [32].

Tabla 4. Aspectos de capacidades analizados en la RSL.

\begin{tabular}{|l|c|c|}
\hline \multicolumn{1}{|c|}{ Capacidades } & Frecuencia & \% \\
\hline Capacidad de innovación & 12 & 60 \\
\hline Capacidad de absorción & 6 & 30 \\
\hline $\begin{array}{l}\text { Capacidad de dirección } \\
\text { estratégica }\end{array}$ & 2 & 10 \\
\hline
\end{tabular}

Fuente: Elaboración propia a partir de la RSL realizada.

La Tabla 4 muestra que las capacidades de innovación $(60 \%)$ es la variable más analizada y estudiada y se define como "la capacidad de la organización para integrar cada uno de los componentes, incorporando el aprendizaje organizacional y la transferencia de conocimiento" [33]. En este sentido, se menciona que las empresas innovadoras exitosas se inclinan por formar portafolios de alianzas estratégicas nacionales e internacionales, para así fortalecer e incrementar la transferencia de conocimiento [34]. Es así como toda la capacidad de innovación de la organización es dedicada a la obtención de financiación y recursos humanos orientados a la investigación y desarrollo $(\mathrm{I}+\mathrm{D})$. Este comportamiento es más frecuente en las IES, ya que aparte de que hay una inversión en I+D, dichas actividades permiten que las organizaciones tengan mayores ganancias y retornos de la inversión, así como un mayor posicionamiento en el mercado.

La segunda variable corresponde a la capacidad de absorción (30\%); esta se define como "la capacidad por parte de otras organizaciones o personas a utilizar, adoptar, replicar, mejorar o modificar la tecnología, habilidades o conocimientos para sus propios fines" [34]. Algunos de los resultados que se pueden evidenciar con la capacidad de absorción son: la adaptación de tecnología, mejora en la transferencia de conocimiento, desarrollo de nuevas capacidades y un conocimiento de los clientes, que puede llevar a que las organizaciones se adapten a las exigencias del mercado y lleguen hacer más innovadores [20]. 
La tercera variable es la capacidad de direccionamiento estratégico (10\%). Se define como la importancia de direccionar estratégicamente a la organización en la toma de decisiones y fortalecer en la misma los intermediarios; la flexibilidad, la apertura y la conectividad de las estructuras de la organización; la participación en redes; la construcción de confianza en las relaciones por medio de la comprensión mutua; aprendizaje de activo de la organización; fortalecimiento de la cooperación debido a la creación de capacidades; y el cambio de cultura [35].

Esto incide para que en la organización haya una transferencia de conocimiento y tecnología permanente al incorporarse en sus diferentes procesos estratégicos [35]. En cuanto a los aspectos colectivos empresariales analizados en la RSL, la tabla 5 muestra que el establecer alianzas estratégicas de investigación y desarrollo (25\%) es la variable más estudiada y analizada. Esta se define como el "mecanismo utilizado por empresas para incrementar la transferencia de conocimiento voluntario" [18].

Este tipo de cooperación demuestra aumentar la capacidad de la empresa para innovar [24] y, a su vez, impulsa una actuación financiera positiva de la empresa [36]. Es importante resaltar que una cartera de alianzas estratégicas de I+D incluye diferentes tipos de asociación (competidores, clientes, proveedores, de la universidad y centros de investigación). Así pues, las alianzas estratégicas de I+D se deben comprender en dos direcciones de transferencia de conocimiento, ya que cada empresa que se involucra en estos procesos es emisora y receptora de conocimiento [19].

La segunda variable corresponde a la innovación y aprendizaje organizacional (25\%). Se define como "la asimilación y comprensión de los conocimientos organizacionales" [33]; desde este enfoque se plantea que los procesos de innovación y aprendizaje organizacional son más importantes que las redes de capital social en el marco de la transferencia de conocimiento integrado. Es importante resaltar que si la organización tiene limitados recursos, se aconseja que invierta en innovación y aprendizaje organizacional para que tenga una mayor eficiencia en la transferencia de conocimiento [33]. Por otra parte, la capacidad de asimilación de los procesos de aprendizaje organizacional permite realizar análisis económicos de la innovación y el cambio
Tabla 5. Aspectos colectivos empresariales (empresa, universidad, Estado).

\begin{tabular}{|l|c|c|}
\hline \multicolumn{1}{|c|}{ Colectivos empresariales } & Frecuencia & \% \\
\hline $\begin{array}{l}\text { Alianzas de investigación y } \\
\text { desarrollo }\end{array}$ & 5 & 25 \\
\hline $\begin{array}{l}\text { Innovación y aprendizaje } \\
\text { organizacional }\end{array}$ & 5 & 25 \\
\hline Inversionistas en CTi & 3 & 15 \\
\hline Producción innovadora & 3 & 15 \\
\hline Expansión de la organización & 1 & 5 \\
\hline $\begin{array}{l}\text { Internacionalización de la } \\
\text { investigación }\end{array}$ & 1 & 5 \\
\hline $\begin{array}{l}\text { Licenciamiento de invenciones } \\
\text { universitarias }\end{array}$ & 1 & 5 \\
\hline Ventaja competitiva & 1 & 5 \\
\hline
\end{tabular}

Fuente: Elaboración propia a partir de la RSL realizada.

tecnológico, así como otros resultados de gestión, por ejemplo, informes de proyectos de investigación, sistemas de medición de rendimiento, productividad y de innovación [30].

La tercera variable corresponde a los inversionistas en $\mathrm{CTi}(15 \%)$. Esta variable se define como "la financiación de los recursos humanos dedicados a la I+D en la organización" [37]. De allí surge también la necesidad de tener diversos actores de $\mathrm{I}+\mathrm{D}$, así como las oportunidades de financiación, esto hace que las organizaciones y sus sistemas de innovación prosperen [31], y que las compras nacionales de tecnología tengan un impacto favorable para la organización a nivel de innovación [38].

La cuarta variable corresponde a la producción innovadora (15\%). Esta se define como "la utilización de nuevas estrategias de investigación y desarrollo dentro de la organización utilizando todos los canales"; en este sentido, las organizaciones han descuidado los proveedores de servicios de innovación externos, denominados "entidades de innovación del contrato" [39]. Estas organizaciones ayudan a motivar el proceso de innovación, con el conocimiento científico y tecnológico para mejorar el desarrollo de nuevos productos [39]. $\mathrm{El}$ incremento de producción innovadora permite también expandir los mercados y adaptarse a los cambios del mismo. Asimismo, es importante tener en cuenta que si en una organización se incentiva la producción innovadora se mejora la flexibilidad, la transferencia de conocimientos a los mercados, 
y finalmente se impulsen las exportaciones en las organizaciones [40].

La quinta variable corresponde a la expansión de la organización (5\%). Dicha variable es definida como "la capacidad de la organización de penetrar otros mercados nacionales e internacionales" [28]; es esta una forma de transferir el conocimiento y realizar alianzas estrategias de $\mathrm{I}+\mathrm{D}+\mathrm{i}$ con otras organizaciones, para poder llevar a cabo la penetración en los mercados internos y externos.

La sexta variable corresponde a la internacionalización de la investigación (5\%). Su definición es: "la importancia de tener canales de comunicación entre los desarrollos de I+D con organismos o entes internacionales, donde se evidencien actividades colaborativas entre profesionales de las áreas interesadas" [35]. Se trata de realizar acuerdos o convenios específicos en materia de I+D+i, donde se puedan visualizar intercambios académicos, pasantías internacionales, investigaciones en conjunto, transferencia del conocimiento de estudiantes y docentes de las organizaciones o IES involucradas, donde haya igual retribución de conocimiento para ambas partes.

La séptima variable corresponde al "licenciamiento de invenciones universitarias". En ella el vendedor de tecnología tendrá un incentivo para "dejar de lado" algunos de los proyectos, elevando así las creencias de los compradores en la calidad esperada; esto se traduce en menos, pero las innovaciones más valiosas que se venden a precios más altos [14].

La octava variable hace referencia a la ventaja competitiva, que de acuerdo con los resultados corresponde a $5 \%$, en donde se relaciona principalmente con el "conocimiento tácito de la empresa (know how)" lo que según los textos analizados se considera una fuente importante para generar ventaja competitiva en donde la empresas se podría consolidar su participación apuntándole a una estrategia global [20].

En definitiva, se puede observar que los resultados y análisis realizados en la RSL posibilitan que se reduzcan los sesgos en los modelos y visiones sistémicas que son expuestas en el estudio del proceso de transferencia de resultados de investigación en las instituciones de educación superior, brindando confiabilidad sobre la pertinencia y la calidad de las referencias bibliográficas utilizadas y las variables incorporadas en dichos análisis.

\section{CONCLUSIONES}

La RSL busca reducir los sesgos presentes en las revisiones subjetivas y permite realizar un proceso auditable, ordenado y reproducible de búsqueda, permitiendo así la valoración crítica de los estudios consultados que responden a las necesidades y preguntas de investigación previamente definidas. Asimismo, dicha información muestra la necesidad de introducir análisis frente a las etapas y características del proceso de transferencia de resultados de investigación. Por consiguiente, es importante el análisis realizado del proceso de transferencia de conocimiento a partir de capacidades de innovación, desde un enfoque integrado, ya que cada aspecto o variable encontrada en la RSL se puede aplicar al contexto de las instituciones de educación superior.

Según la información analizada de la RSL para poder tener buenos procesos de transferencia se debe llevar a cabo alianzas estratégicas, alianzas de investigación y desarrollo, expansión en el mercado, conocimientos sobre competidores, innovación y aprendizaje organizacional, conocimiento sobre los clientes, inversión en CTi, y acciones de apropiación de conocimiento en las organizaciones.

Por otro lado, la dificultad para encontrar un mayor número de revistas indexadas de calidad que permitieran aplicar el diseño de la RSL da cuenta del bajo impacto que actualmente la temática de transferencia de resultados de investigación tiene para ser incorporada las principales publicaciones científicas a nivel mundial. Esto refleja que aún existen deficiencias en la visibilidad y difusión de las investigaciones orientadas a resolver dicha problemática, desde el término de transferencia de resultados de investigación, esto debido a que el $80 \%$ de los artículos indexados encontrados en la RSL hablan de los términos transferencia de conocimiento y transferencia de tecnología, reforzando la necesidad de indagar e investigar más acerca de la transferencia de resultados de investigación en las IES.

Adicionalmente, los datos arrojados de la población analizada por los artículos científicos indican que aun el enfoque de análisis está centrado en $45 \%$ en la 
empresa; aunque los procesos de las organizaciones son parecidos y se pueden adaptar a las IES, resulta imperativo que se fomente la investigación desde el aporte de las IES y sus procesos organizacionales internos, incluyendo en este aspecto las capacidades de innovación.

Entre las razones que pueden explicar la escasez de publicaciones en esta temática específica, se resaltan las siguientes: falta de acceso y apoyo a la investigación longitudinal, menor número de académicos capacitados para abordar el proceso investigativo acerca del tema de transferencia de conocimientos y capacidades de innovación, la falta de inclusión de OTT/OTRIS en las organizaciones donde se pueda gestionar, administrar y comercializar la transferencia de conocimiento.

\section{REFERENCIAS}

[1] M. Becerra. "La transferencia de tecnología en Japón. Conceptos y enfoques". Cienc. UANL. 2014; 2 (1): 6-50.

[2] Rodríguez Orejuela A. Transferencia de conocimiento en relaciones interorganizacionales: su efecto sobre el desempeño de la firma receptora. Estud. Gerenciales [Internet]. 2007; 23 (103): 13-37. Available from: http://www.sciencedirect.com/science/ article/pii/S0123592307700086

[3] V.J.R., G.C.L., L. W.Z., V.J.D.P. Desarrollo de una Metodología de Evaluación de Capacidades de Innovación. Perf. Coyunt. Económica [Internet]. 2010;(15):133-48. Available from: http://aplicacionesbiblioteca.udea.edu.co:2082/ doaj$?$ func $=$ search\&template $=\&$ uiLanguage $=$ en\&query=Antioquia+AND+cluster+OR+A ntioquia+AND+Innovacion

[4] Etzkowitz H. Innovation in innovation: the Triple Helix of university-industrygovernment relations. Soc Sci Inf Sur Les Sci Soc [Internet]. 2003;42:293-337. Available from: //000185234300002

[5] Arias-Pérez J.E., Aristizabal-Botero C.A. Transferencia de conocimiento orientada a la innovación social en la relación cienciatecnología y sociedad. Rev Científica Pensam. y Gestión. 2011; (31): 1-13.

[6] Campos E.B. La Tercera Misión de la Universidad: El reto de la Transferencia del conocimiento. Rev madri+ d. (41): 2-50.
[7] Jaramillo, M.A., Arango, O.G., Cárdenas, L.Z., Llanos, R.A., Pico, J.C., G.M.G. et al. Un Análisis de La Transferencia y Apropiación del Conocimiento en la Investigación de Universidades Colombianas. Investig. y Desarro. [Internet]. 2005; 13 (1): 128-9. Available from: http://search.ebscohost. com/login.aspx?direct $=$ true $\& d b=a 9 h \& A N$ $=21760326 \&$ lang $=$ es \&site $=$ ehost-live

[8] Guerrero M., Urbano D. Knowledge and Technology Transfer Strategies: Best Practices in Spanish Entrepreneurial Universities. Gest. y Polit Publica. 2012; 21 (1): 107-39.

[9] Petticrew M., Roberts H. Systematic Reviews in the Social Sciences: A Practical Guide [Internet]. Cebma.Org. 2006. 336 p. Available from: xa.yimg.com/kq/groups/ 18751725/462617161/name/manalysis.pdf? nhttp://www.cebma.org/wp-content/uploads/ Pettigrew-Roberts-SR-in-the-Soc-Sc.pdf nhttp://books.google.com.br/books/about/ Systematic_Reviews_in_the_Social_Science. html?id=_Ly3aPhTkbkC\&redir_esc $=y$

[10] Valencia Arias A. Aproximación a la dinámica y complejidad del emprendimiento desde los sistemas. 2012;148. Available from: http:// www.bdigital.unal.edu.co/9091/

[11] Kitchenham B. Procedures for performing systematic reviews. Keele, U.K., Keele Univ [Internet]. 2004; 33 (TR/SE-0401): 28. Available from: http://csnotes.upm.edu.my/kelasmaya/ pgkm20910.nsf/0/715071a8011d4c2f4825 77a700386d3a/\$FILE/10.1.1.122.3308[1]. pdf \nhttp://tests-zingarelli.googlecode. com/svn-history/r336/trunk/2-Disciplinas/ MetodPesquisa/kitchenham_2004.pdf

[12] Guerra, J.A., Muñoz, P.M. \& Santos, J. Las revisiones sistemáticas, niveles de evidencia y grados de recomendación. 2003.

[13] Elsevier. Scopus. 2014; Available from: http:// www.americalatina.elsevier.com/corporate/ es/scopus.php

[14] Macho-Stadler, I., Pérez-Castrillo, D., Veugelers, R. Licensing of university inventions: The role of a technology transfer office. Int J Ind Organ. 2007; 25 (3): 483-510.

[15] SCImago. SCImago Journal \& Country Rank. 2014; Available from: http://www. scimagojr.com/aboutus.php

[16] Leischnig, A., Geigenmueller, A., Lohmann, S. On the role of alliance management capability, 
organizational compatibility, and interaction quality in interorganizational technology transfer. J Bus Res [Internet]. Elsevier Inc.; 2014; 67 (6): 1049-57. Available from: http:// dx.doi.org/10.1016/j.jbusres.2013.06.007

[17] Che Rose, R., Uli, J., Kumar, N., Abdul Wahab S. A Review on the Effects of Interfirm Technology Transfer Characteristics and Degree of Technology Transfer. Eur J Soc Sci. 2009; 8 (2): 297-309.

[18] Duysters, G., Lokshin, B. Determinants of alliance portfolio complexity and its effect on innovative performance of companies. J Prod Innov Manag. 2011; 28 (4): 570-85.

[19] Schulze, A., Brojerdi, G., Von Krogh, G. Those who know, do. Those who understand, teach. Disseminative capability and knowledge transfer in the automotive industry. J Prod Innov Manag. 2014; 31 (1): 79-97.

[20] Montazemi, A.R., Pittaway, J.J., Qahri, Saremi H., Wei, Y. Factors of stickiness in transfers of know-how between MNC units. J Strateg Inf Syst [Internet]. Elsevier B.V.; 2012; 21 (1): 31-57. Available from: http:// dx.doi.org/10.1016/j.jsis.2012.01.001

[21] Nelson, R.R., Winter, S.G. An evolutionary theory of economic change. Cambridge MA Belknap. 1982. 437 p.

[22] Cohen, W.M., Levinthal, D. a. Absorptive Capacity: A New Perspective on and Innovation Learning. Adm Sci Quaterly. 1990; 35 (1): 128-52.

[23] Grimpe, C., Hussinger, K. Formal and Informal Technology Transfer from Academia to Industry: Complementarity Effects and Innovation Performance [Internet]. Technology. Taylor \& Francis; 2008. p. 1-28. Available from: <Go to ISI $>$ ://WOS:000327585200001। nhttp://ssrn.com/paper=1283685

[24] Lichtenthaler, U. Open innovation and the importance of actively managing outward technology transfer. Int J Technol Intell Plan [Internet]. 2008; 4 (4): 466-79. Available from: http://www.inderscience.com/offer. php?id=20875

[25] Ranga, M., Temel, S., Ar, I.M., Yesilay, R.B., Sukan, F.V. Building Technology Transfer Capacity in Turkish Universities: A critical analysis. Eur J Educ. 2016; 51(1): 90-106.

[26] Zhang, G., Duan, H., Zhou, J. Investigating determinants of inter-regional technology transfer in China: a network analysis with provincial patent data. Rev Manag Sci. 2016; 10 (2): 345-64.

[27] De Prá Carvalho, A., Dos Reis, D.R., De Matos, E.A.A., Carvalho, S. Mechanisms of technology transfer centre of information technology for the southwest region of Parana - Brazil. 2014;10 (2): 99-108.

[28] Idris, A., Tey, L.S. Exploring the motives and determinants of innovation performance of Malaysian offshore international joint ventures. Manag Decis. 2011; 49 (10): 1623-41.

[29] Villalobos, B., Ovallos, D., Maldonado, D. $\&$ De la Hoz, S. Factores que inciden en el desempeño eficiente de una Oficina de Transferencia de Resultados de Investigación(OTRI): Caso Cientech. Espacios. 2016; 37 (9): 10.

[30] Molero, J. La Transferencia de Tecnología revisitada: conceptos básicos y nuevas reflexiones a partir de un modelo de gestión de excelencia. ARBOR Ciencia, Pensam. y Cult. 2008; 732: 637-51.

[31] Bienkowska, D., Larsen, K., Sörlin, S. Publicprivate innovation: Mediating roles and ICT niches of industrial research institutes Mediating roles and ICT niches of industrial research institutes. Innov Manag Policy Pract. 2010; (12): 206-16.

[32] Colciencias. Modelo de medición de grupos de investigación, desarrollo tecnológico o de innovación y reconocimiento de investigadores del sistema nacional de ciencia, tecnología e innovación. País: Departamento Administrativo de Ciencia, Tecnología e Innovación. 2014; Available from: http://www. colciencias.gov.co/sites/default/files/upload/ documents/documento_medicion_grupos_-investigadores_version_final_15_10_2014.pdf.

[33] Rhodes, J., Lok, P., Hung, R.Y.-Y., Fang, S.-C. An integrative model of organizational learning and social capital on effective knowledge transfer and perceived organizational performance. J Work Learn [Internet]. 2008; 20 (4): 245-58. Available from: http://search. proquest.com.ezproxy.usherbrooke.ca/ abicomplete/docview/198435149/DE63535 A28624323PQ/53?accountid=13835 \nhttp:// media.proquest.com.ezproxy.usherbrooke. ca/media/pq/classic/doc/1484477901/fmt/ pi/rep/NONE?hl=,knowledge+transfer\&ci t:auth=Rhodes. 
[34] Bohlmann, J.D., Calantone, R.J., Zhao, M. The effects of market network heterogeneity on innovation diffusion: An agent-based modeling approach. J Prod Innov Manag. 2010; 27 (5): 741-60.

[35] Johnston, L., Robinson, S. \& Lockett N. Recognising "open innovation" in HEI-industry interaction for knowledge transfer and exchange. Int J Entrep Behav Res. 2010; 16 (6): 540-60.

[36] Fernandes, C.I., Ferreira, J.J.M. Knowledge spillovers: Cooperation between universities and KIBS. R.D. Manag. 2013; 43 (5): 461-72.

[37] Torkkeli, M.T., Podmetina, D., Kojola, A.M.Y., Vaatanen J. Knowledge absorption in an emerging economy - the role of foreign investments and trade flows in Russia. Int J Bus Excell [Internet]. 2009; 2 (3/4): 269. Available from: http://www. scopus.com/inward/record.url?eid=2-s2.066249097068\&partnerID=tZOtx3y1

[38] Li, X. (2011). Sources of External Technology, Absorptive Capacity, and Innovation Capability in Chinese State-Owned HighTech Enterprises. World Development, 39 (7), 1240-1248.

[39] Saur-Amaral, I. \& Amaral, P. (2010). Contract innovation organisations in action: Doing collaborative new product development outside the firm. International Journal of Technology Intelligence and Planning, 6 (1), 42-62.

[40] Rodriguez, C.M., Wise, J.A., \& Martínez, C.R. (2013). Strategic capabilities in exporting: An examination of the performance of Mexican firms. Management Decision, 51 (8), 1643-1663. 